\title{
As três tsunamis da Psiquiatria: mau uso, abuso e não-uso
}

(continued) Assessment Scales and Diagnostic Criteria: How to Use them Properly

\author{
Clarice Gorenstein ${ }^{1}$ \\ Laura H. S. G. DE ANDrade ${ }^{2}$
}

O poder das ondas destrutivas é muito grande e suas conseqüências, avassaladoras. Assim como na natureza, as tsunamis podem atingir todas as áreas do conhecimento, entre elas a Psiquiatria, como bem apontou o Dr. Guido Arturo Palomba.

De fato, os instrumentos diagnósticos, os exames subsidiários e as escalas de avaliação podem transformar-se em tsunamis extremamente prejudiciais para a clínica e para a pesquisa, quando submetidas ao mau uso por profissionais não treinados, que desconhecem seus conceitos subjacentes ou que ultrapassam, inadvertidamente, seus limites.

A Psiquiatria destaca-se das outras especialidades médicas por explorar o mundo fenomenológico das experiências mentais, temperamento, capacidades intelectuais e comportamentos variados. A tarefa do psiquiatra, em última análise, é reconhecer aqueles sintomas que necessitam de atenção médica. A anamnese do profissional de saúde mental é embasada nos seus conhecimentos teóricos e prática clínica, que determinarão sua formulação diagnóstica e conduta terapêutica. Nada a substitui. Apesar disso, atualmente são modernos os manuais diagnósticos como a CID-10 e do DSM-IV. Por que precisamos encontrar critérios operacionais para fenômenos psicológicos? Ou, ainda, para que mensurá-los em um contínuo de intensidade ou freqüência? Será, como sugere o Dr. Palomba, para serem usados como "testezinhos de revistas populares para meninas adolescentes"?

Contrastando com outras especialidades médicas, nas quais estão disponíveis recursos diagnósticos subsidiários objetivos, a ausência de marcadores biológicos consistentes faz com que a maioria dos diagnósticos psiquiátricos baseie-se na avaliação subjetiva da presença de sintomas e síndromes clínicas. Há maior grau de concordância entre diferentes investigadores, quando a atribuição do diagnóstico é orientada por critérios operacionais e entrevistas psiquiátricas padronizadas, possibilitando à comunicação internacional e à comparação de resultados obtidos em diferentes centros. Essa necessidade tornou-se mais evidente a partir da introdução dos primeiros psicofármacos, uma vez que, eram observadas acentuadas variações de diagnóstico quando diferentes psiquiatras atribuíam diagnósticos clínicos a um mesmo grupo de pacientes.

Há mais de 30 anos, estudos clássicos mostraram que pacientes diagnosticados como esquizofrênicos e alcoólatras em Nova York foram diagnosticados como

Recebido: 23/02/2005

1 Professora Associada, Departamento de Farmacologia do Instituto de Ciências Biomédicas da Universidade de São Paulo (USP); LIM-23 Laboratório de Psicofarmacologia do Instituto de Psiquiatria do Hospital das Clínicas da Faculdade de Medicina da USP (Ipq-HC-FMUSP).

2 Doutora em Psiquiatria pela Faculdade de Medicina da Universidade de São Paulo (FMUSP); Coordenadora do Núcleo de Epidemiologia do Instituto de Psiquiatria, do Hospital das Clínicas da Faculdade de Medicina da USP (Ipq-HC-FMUSP).

Endereço para correspondência: LIM 23, Departamento de Psiquiatria da Faculdade de Medicina da Universidade de São Paulo (IPq-FMUSP). Rua Ovídio Pires de Campos, 785, térreo 05403-010 - São Paulo - SP. Fone: (11) 3069-6950; e-mail: cgorenst@usp.br 
sofrendo de mania e depressão psicótica em Londres (Cooper et al., 1972). As principais causas da variação no diagnóstico clínico deveram-se às diferenças dos critérios adotados e à forma de se obter as informações dos indivíduos avaliados. $\mathrm{O}$ desenvolvimento de critérios diagnósticos operacionais e entrevistas psiquiátricas padronizadas teve como objetivo aumentar a concordância entre diferentes investigadores e permitir comparações de resultados, possibilitando investigações sobre curso e prognóstico e sobre a eficácia de diferentes tratamentos, de acordo com particularidades dos pacientes (por exemplo, no caso de mulheres dependentes de álcool com depressão comórbida ou em pacientes com transtorno bipolar em comorbidade com transtorno obsessivo-compulsivo).

As escalas utilizadas em psicofarmacologia têm como objetivo mensurar a intensidade dos sintomas para avaliar sua evolução em função de uma determinada intervenção terapêutica. Aintrodução de novos psicofármacos baseia-se nos resultados de ensaios clínicos, que utilizam tais instrumentos para corroborar sua eficácia terapêutica. Como, na atribuição de escores, sempre existe uma parcela de subjetividade, tanto do paciente quanto do entrevistador e, considerando que muitos dos sintomas dos transtornos psiquiátricos são inerentemente subjetivos, essas "medidas" devem ser consideradas como estimativas aproximadas.

O número de instrumentos diagnósticos e de escalas de avaliação de sintomas disponíveis é muito grande e novos instrumentos são constantemente desenvolvidos. Vários princípios teóricos regem a elaboração das escalas e o conhecimento das propriedades psicométricas desses instrumentos facilita a escolha da mais adequada para cada finalidade. A psicometria tornou-se uma área de estudo própria e sofisticada. Entre as propriedades atribuídas às escalas de avaliação, destacam-se a confiabilidade, que se refere à capacidade de detectar um escore verdadeiro em relação ao erro causado pela imprecisão na medida; e a validade, que representa a capacidade do instrumento de medir o que ele se propõe a medir, respeitando inclusive as variações etnoculturais, observadas nas manifestações psicopatológicas. Um dos aspectos da validade envolve a comparação do instrumento com um critério externo já existente e considerado como "padrão ouro", que na maioria das vezes é o diagnóstico clínico.

O Brasil também contribuiu para o progresso da psicometria: há 20 anos foi realizada a primeira reunião, tendo como tema "Instrumentos e critérios para diagnósticos psiquiátricos em pesquisas com psicofármacos". Hoje dispomos de centros especializados no estudo e treinamento de instrumentos diagnósticos (Gentil Filho et al., 1986; Quintana et al., 2004). O livro "Escalas de Avaliação Clínica em Psiquiatria e Psicofarmacologia" (Gorenstein et al., 2000) apresenta a experiência e o conjunto de dados brasileiros, reunindo estudos de tradução, adaptação, validação e desenvolvimento das principais escalas de avaliação clínica habitualmente utilizadas em Psiquiatria e Psicofarmacologia.

Embora não vise substituir o especialista, a mensuração em Psiquiatria gera grande dicotomia: os que a aceitam, mesmo reconhecendo suas limitações e críticas, e os que a repudiam convictamente. Para estes últimos, as avaliações padronizadas são simplistas, pois, transformam uma dimensão clínica em um número ou categoria, relegando a segundo plano o julgamento clínico subjetivo.

Vale lembrar que qualquer sistema classificatório, escala de avaliação ou entrevista diagnóstica nada mais é do que uma teoria (um conceito) e uma tecnologia (um instrumento operacional). Ou seja, o clínico que faz sua avaliação de forma não padronizada, baseando-se na sua experiência profissional e impressão pessoal, também está utilizando o mesmo referencial teórico que gerou as entrevistas estruturadas ou semi-estruturadas.

Quanto aos exames subsidiários, especialistas em neuroimagem reconhecem que a Psiquiatria tem se beneficiado dos avanços tecnológicos que aumentaram a nitidez das imagens do cérebro humano, tanto para a investigação de aspectos anatômicos estruturais quanto funcionais. Com esses métodos, têm sido demonstradas alterações em áreas cerebrais especí ficas em diversos transtornos mentais. Entretanto, até o momento não há aplicações práticas para a neuroimagem no diagnóstico diferencial de transtornos psiquiátricos. Os resultados das pesquisas são quase sempre fruto de comparações estatísticas de médias de medidas cerebrais entre grupos de pacientes e controles normais. Quando as imagens são avaliadas individualmente, são detectadas anormalidades em apenas uma parcela dos pacientes e há considerável variabilidade quanto à natureza e à localização cerebral. Portanto, as imagens não têm sensibilidade e especificidade suficientes a ponto de tornar possível estabelecer uma ligação definitiva com a clínica (Rocha et al., 2001). Como as técnicas de neuroimagem ainda não foram incorporadas à rotina clínica em Psiquiatria, é difícil entender como "a emissão de pósitrons, a ressonância magnética, o expert-system e outros assemelhados dizimaram a semiologia psiquiátrica", como sugere o Dr. Palomba.

A existência de um componente arbitrário é inerente a qualquer instrumento de avaliação em Psiquiatria, assim como, na Medicina em geral, e trata-se de uma limitação intransponível.

Já o mau uso é comum nos profissionais sem treinamento suficiente no emprego dos instrumentos ou, ainda, sem formação fenomenológica consistente. Essas tsunamis podem gerar erros diagnósticos e interpretações imprecisas, que repercutirão no tratamento e prognóstico dos pacientes com transtornos mentais. 
No entanto, podemos também nos questionar se, em qualquer área da Medicina, seria aceitável ignorar os avanços terapêuticos e metodológicos, tornando a prática - mesmo que inadvertidamente reflexo de uma ideologia em detrimento do benefício do paciente. Atualmente, seria possível oferecer uma terapêutica adequada, com menor perfil de efeitos colaterais, sem se beneficiar dos estudos, escalas e critérios diagnósticos?

As tsunamis talvez não sejam apenas fruto da imbecilidade, como apontado pelo Dr. Guido Palomba, mas, principalmente, da estagnação do conhecimento.

\section{Referências bibliográficas}

Cooper, J.E.; Kendell, R.E.; Gurland, B.J.; Sharpe, L.; Copeland, J.R.M.; SIMON, R. - Psychiatric diagnosis in New York and London. Oxford University Press, London, 1972.

Gentil Fllho, V.; Andrade, L.H.G.; Lotufo Neto, F. - PSE: tradução, treinamento e limitações ao seu uso no Brasil. Rev ABPAPAL, 8 (supl.): 30-33, 1986.

Gorenstein, C.; ANDRADE, L.H.S.G.; Zuardi, A.W. (EDS.) - Escalas de Avaliação Clínica em Psiquiatria e Psicofarmacologia, Lemos Editorial, São Paulo, 2000, pp. 438.
Quintana, M.l.; Andreoli, S.B.; Jorge, M.R.; Gastal, F.L.; Miranda, C.T. - The reliability of the Brazilian version of the Composite International Diagnostic Interview (CIDI 2.1). Braz J Med Biol Res 37(11):1739-45, 2004.

Rocha, E.T.; Alves, T.C.T.F.; GarRido, G.E.J.; Buchpiguel, C.A.; Nitrini, R.; Busatto Filho, G. - Novas técnicas de neuroimagem em psiquiatria: qual o potencial de aplicações na prática clínica? Rev Bras Psiquiatr 23 (Suppl.1):58-60, 2001. 